Walter Bitterlich | Sabine Ausmeier | Ulrich Lohmann

Gasturbinen und Gasturbinenanlagen 
Numerische Beanspruchungsanalyse von Rissen

von M. Kuna

Einführung in die Festigkeitslehre

von V. Läpple

Mechanisches Verhalten der Werkstoffe

von J. Rösler, H. Harders und M. Bäker

Technologie der Werkstoffe

von J. Ruge und $\mathrm{H}$. Wohlfahrt

Ermüdungsrisse

von H. A. Richard und M. Sander

Werkstoffkunde

von W. Weißbach

Aufgabensammlung Werkstoffkunde und Werkstoffprüfung von W. Weißbach und M. Dahms

Mechanisches Verhalten der Werkstoffe von J. Rösler

Kunststoffe in der Ingenieuranwendung von M. Bonnet 
Walter Bitterlich | Sabine Ausmeier

Ulrich Lohmann

\section{Gasturbinen und Gasturbinenanlagen}

Darstellung und Berechnung

Mit 143 Abbildungen, 60 Tabellen

und ausführlichen Berechnungsbeispielen

STUDIUM

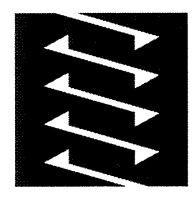


Bibliografische Information der Deutschen Nationalbibliothek Die Deutsche Nationalbibliothek verzeichnet diese Publikation in der Deutschen Nationalbibliografie; detaillierte bibliografische Daten sind im Internet über $<$ http://dnb.d-nb.de> abrufbar.

Prof. Dr.-Ing. Walter Bitterlich ist Professor für Berechnungsmethoden in der Energietechnik am Lehrstuhl für Energie- und Kraftwerkstechnik an der Universität Essen.

Dr.-Ing. Sabine Ausmeier ist wissenschaftliche Mitarbeiterin am Lehrstuhl für Energie- und Kraftwerkstechnik an der Universität Essen.

Dipl.-Ing. Ulrich Lohmann ist wissenschaftlicher Mitarbeiter am Lehrstuhl für Energie- und Kraftwerkstechnik an der Universität Essen.

1. Auflage 2002

Alle Rechte vorbehalten

(c) Vieweg+Teubner Verlag | Springer Fachmedien Wiesbaden GmbH 2002

Lektorat: Thomas Zipsner | Ellen Klabunde

Vieweg+Teubner Verlag ist eine Marke von Springer Fachmedien.

Springer Fachmedien ist Teil der Fachverlagsgruppe Springer Science+Business Media.

www.viewegteubner.de

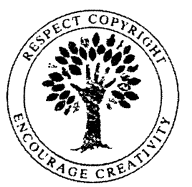

Das Werk einschließlich aller seiner Teile ist urheberrechtlich geschützt. Jede Verwertung außerhalb der engen Grenzen des Urheberrechtsgesetzes ist ohne Zustimmung des Verlags unzulässig und strafbar. Das gilt insbesondere für Vervielfältigungen, Übersetzungen, Mikroverfilmungen und die Einspeicherung und Verarbeitung in elektronischen Systemen.

Die Wiedergabe von Gebrauchsnamen, Handelsnamen, Warenbezeichnungen usw. in diesem Werk berechtigt auch ohne besondere Kennzeichnung nicht zu der Annahme, dass solche Namen im Sinne der Warenzeichen- und Markenschutz-Gesetzgebung als frei zu betrachten wären und daher von jedermann benutzt werden dürften.

Umschlaggestaltung: KünkelLopka Medienentwicklung, Heidelberg

Gedruckt auf säurefreiem und chlorfrei gebleichtem Papier 


\section{Vorwort}

Als Ältester der drei Autoren beschäftige ich mich mit Gasturbinen und ihren Komponenten schon mehr als 30 Jahre an den Hochschulen in Aachen und Essen. Zunächst in der Forschung später auch in der Lehre.

Vertieft wurde die Beziehung vor einigen Jahren durch mehrere Forschungsvorhaben zu diesem Thema, die wir zusammen mit einem Hersteller durchführten. Dabei reifte der Entschluss, die gesammelten Erfahrungen in einem Buch zusammenzutragen. Mit meinen beiden - viel jüngeren - Mitautoren, Frau Sabine Ausmeier und Herrn Ulrich Lohmann, haben wir ein Buch geschrieben, das das Verständnis für Gasturbinen vor allem über die physikalischen und formelmäßigen Zusammenhänge und die daraus folgende Berechnung vertiefen soll.

Obwohl natürlich in den Zahlen und den speziellen Anordnungen der jetzige Stand der Technik dargestellt wird, soll das Buch auch in die Zukunft „hineinreichen“. Es richtet sich eigentlich an alle, die mehr über Gasturbinen, auch in der "modernen" Form als GuD-Kombi-Anlage, wissen wollen.

$\mathrm{Zu}$ danken ist an dieser Stelle einer Reihe von Mitarbeitern und Studenten, die Beiträge zum Buch geleistet haben. $\mathrm{Zu}$ nennen sind: A. Baars, C. Bernhardt, A. Göb, L. Derichs, K. Divivier, Ch. Engel, O. Hörstgen, H.P. Marschall, M. Odrost und D. Wellié. Zu danken ist natürlich auch dem Verlag, der das „finanzielle Wagnis“ der Herausgabe eines wissenschaftlichen Buches eingegangen ist.

Essen, am 15. April 2002

Walter Bitterlich 


\section{Inhaltsverzeichnis}

1 Einleitung 1

1.1 Einleitung und Überblick . . . . . . . . . . . . . . . . . 1

1.2 Historische Entwicklung . . . . . . . . . . . . . . . 4

2 Grundlagen $\quad 7$

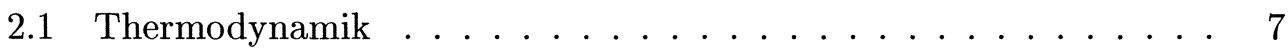

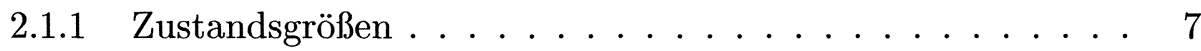

2.1.2 Zustandsgleichungen . . . . . . . . . . . . . 8

2.1.3 Zustandsänderungen . . . . . . . . . . . . . . . . 19

2.2 Strömungsmechanik . . . . . . . . . . . . . . 26

2.3 Verbrennungslehre . . . . . . . . . . . . . . . . 34

2.4 Wärmeübergang . . . . . . . . . . . . . . . . 40

2.4.1 Totaltemperatur als Bezugsgröße . . . . . . . . . . 40

2.4.2 Gleich- und Gegenstrom-Wärmeaustauscher . . . . . . . . . 43

2.4 .3 Zirkularität . . . . . . . . . . . . . . . 48

2.4.4 Grundfälle für den Wärmeübergang . . . . . . . . . . 50

2.4.5 Abschätzen der Größe eines Wärmeaustauschers . . . . . . 53

2.5 Wärmestrahlung . . . . . . . . . . . . . 55

2.6 Strömungswirkungsgrade . . . . . . . . . . . . . . 59

3 Thermische Strömungsmaschinen $\quad 63$

3.1 Stufe einer Strömungsmaschine . . . . . . . . . . . . . 63

3.2 Berechnung der Schaufelprofile . . . . . . . . . . . . . 72

3.2 .1 Skelettlinie . . . . . . . . . . . . . . . 73

3.2 .2 Dickenverteilung . . . . . . . . . . . . 76

3.2.3 Bestimmung der gekrümmten Profilkontur . . . . . . . . . 78

3.2 .4 Gesamtprofilgrößen . . . . . . . . . . . . . . . . . . 79

3.3 Axialverdichter . . . . . . . . . . . . . . . . 81

3.3 .1 Verdichterstufe . . . . . . . . . . . . . 82

3.3.2 Grenzwerte bei der Auslegung . . . . . . . . . . . . . . 91 
3.3 .3 Gesamtverdichter . . . . . . . . . . . . . . 97 97

3.4 Axialturbine . . . . . . . . . . . . . . . . . . . . 97

3.4.1 Turbinenstufe . . . . . . . . . . . . . . 998

3.4 .2 Gesamtturbine . . . . . . . . . . . . . 135

3.4 .3 ISO-Werte der Turbine . . . . . . . . . . . . . . 145

4 Brennkammer $\quad 149$

4.1 Verbrennungsraum . . . . . . . . . . . . . . . . . . 150

4.2 Brennkammer-Diffusor und Brennkammer-Beschleunigungsteil . . . 153

5 Äußere Komponenten 157

5.1 Einlass . . . . . . . . . . . . . . . . . . . . . . 157

5.2 Turbinen-Diffusor . . . . . . . . . . . . . . . . . . 159

5.3 Anlagen Auslass . . . . . . . . . . . . . . . . 159

6 Gesamtauslegung und Optimierung 161

6.1 Schaltungen . . . . . . . . . . . . . . . . . 161

6.2 Berechnung der Gesamtauslegung . . . . . . . . . . . . . . 163

6.3 Energetische Optimierung . . . . . . . . . . . . . . . . . . . . . 164

6.4 Quasi-analytische Berechnung . . . . . . . . . . . . . 167

6.4.1 Quasi-analytische Berechnung einer einfachen Gasturbinenanlage mit Luftkühlung der Turbine . . . . . . . . . 170

7 Wirtschaftlichkeit $\quad 177$

7.1 Kosten . . . . . . . . . . . . . . . . . . . . . . 177

7.1 .1 Fixe Kosten . . . . . . . . . . . . . . . . . . 177

7.1 .2 Brennstoffkosten . . . . . . . . . . . . . . . 181

7.1 .3 Zusatzkosten . . . . . . . . . . . . . . . . . 182

7.1.4 Spezifische Stromgestehungskosten . . . . . . . . . 182

8 Betriebsverhalten $\quad 185$

8.1 Stationäres Betriebsverhalten . . . . . . . . . . . . . . 185

8.1 .1 Einlass . . . . . . . . . . . . . . . . . . . 191

8.1 .2 Verdichter . . . . . . . . . . . . . . . . 193

8.1 .3 Brennkammer . . . . . . . . . . . . . . . 200

8.1 .4 Turbine . . . . . . . . . . . . . . . 202

8.1.5 Diffusor und Auslass . . . . . . . . . . . . . . . 205

8.1 .6 Gesamtanlage . . . . . . . . . . . . . 206

8.2 An- und Abfahren . . . . . . . . . . . . . . . 210

8.2 .1 Anfahren . . . . . . . . . . . . . . 211 


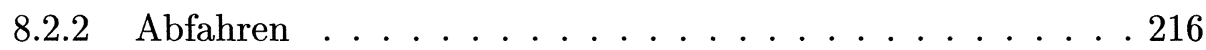

9 Der Dampfteil von Kombinations-Gasturbinenanlagen 219

9.1 Abhitzedampferzeuger . . . . . . . . . . . . . . . 220

9.1.1 Eindruck-Abhitzedampferzeuger . . . . . . . . . 220

9.1.2 Zweidruck-Abhitzedampferzeuger . . . . . . . . . . . 227

9.1.3 Dreidruck-Abhitzedampferzeuger . . . . . . . . . . . . 230

9.2 Investitionskosten des Dampfteils . . . . . . . . . . . . . . . 230

9.2.1 Optimierung einer GuD-Anlage . . . . . . . . . . . 236

A Berechnungsbeispiele Gasturbine $\quad 239$

A.1 Adiabate Gesamtturbine . . . . . . . . . . . . . . . . . . . . . 239

A.2 Konvektionskühlung eines Leitrades . . . . . . . . . . . . . 244

A.3 Filmkühlung einer Leitschaufel . . . . . . . . . . . . . . . . 250

B Ergebnisse der Auslegungsrechnung für eine $30 \mathrm{MW}$ Gasturbinenanlage 255

B.1 Hauptauslegungsdaten . . . . . . . . . . . . . . . . . . . 255

B.2 Komponenten der Anlage . . . . . . . . . . . . . . 256

B.2.1 Einlass . . . . . . . . . . . . . . . . . 256

B.2.2 Verdichter . . . . . . . . . . . . 257

B.2.3 Brennkammer . . . . . . . . . . . . . 265

B.2.4 Turbine . . . . . . . . . . . . . . . 268

B.2.5 Diffusor und Auslass . . . . . . . . . . . . . . . . . . 282

B.3 Gesamtdaten der Anlage . . . . . . . . . . . . . . . . . . . 283

C Ergebnisse für eine GuD-Anlage 287

C.1 Hauptauslegungsdaten für die Berechnung . . . . . . . . . . . . 287

C.1.1 Daten für die Wirtschaftlichkeitsberechnung . . . . . . . . . 289

C.1.2 Gesamtbeurteilungsdaten der GuD-Anlage . . . . . . . . 290

$\begin{array}{lr}\text { Formelzeichen } & 299\end{array}$

$\begin{array}{ll}\text { Literaturverzeichnis } & 307\end{array}$

$\begin{array}{ll}\text { Bildverzeichnis } & 311\end{array}$

$\begin{array}{ll}\text { Tabellenverzeichnis } & 317\end{array}$

$\begin{array}{ll}\text { Stichwortverzeichnis } & 320\end{array}$ 